\title{
Possible classification of pork products by ICP-MS
}

\author{
Motoki GodA $^{* \dagger}$ Member, $\quad$ Yohichiro KojIma ${ }^{*}$ Member
}

(Received February 18, 2019, revised April 15, 2019)

\begin{abstract}
Recently, repeated food adulteration cases have been observed; therefore, ensuring food safety has become essential. First, we examined the possibility of classifying pork products in this study. Second, we considered the practicality of measuring inorganic components of pork products via inductively coupled plasma mass spectrometry (ICP-MS). Additionally, we investigated whether classification collation using a neural network is possible by learning the inorganic components measured by ICP-MS. Classification collation of pork products was found to be possible for each category (product type, product part, and cooking method) using the inorganic component contents measured by ICP-MS.
\end{abstract}

Keywords: Pork product, Classification, Chemical components, Neural network, ICP-MS,

\section{Introduction}

To protect food safety, ingredient evaluation has been carried out by numerous chemical analysis methods. Recently, with various concerning factors such as increased food importation, production, fraud, and poisoning, it is more urgent than ever to ensure safety. From the background mentioned above, it is increasingly important to quickly match and accurately classify analysis results for many foods.

Conventionally, various chemical component contents have been quantified using chemical analyzers, such as atomic absorption spectrometry, fluorescent X-ray diffractometry, gas chromatography, mass spectrometry, and highperformance liquid chromatography. However, quantitative data collected by these methods is used from a nutritional aspect and are not for classification and identification of food types and forms, where consideration of its importance is increasing absent [1] [3]. Therefore, the study group examined optimization and facilitation possibilities for food classification using the chemical substance content of food quantified and converted by quantitative chemical methodology procedures [4] [13].

In this report, we examined whether classification of pork products into categories-product type, product part, and cooking method-is possible using 11 inorganic components-sodium, potassium, calcium, magnesium, phosphorus, iron, zinc, copper, iodine, selenium, and chromium-quantified by ICP-MS. For the examination, we used the neural network method and provided a preliminary results report and future development plans.

\footnotetext{
*Corresponding: gouda@njpc.co.jp

$\dagger$ Hokkaido University of Science

7-Jo 15-4-1, Maeda, Teine, Sapporo, Hokkaido, Japan 006-858

$\doteqdot$ North Japan Port Consultants Co., Ltd

S2E2, 8-1, Sapporo, Hokkaido, Japan 060-0052
}

\section{Materials and method}

2.1 Pork products as subject data This study was conducted on 88 pork product types identified by the Ministry of Education, Culture, Sports, Science, and Technology. Using the 11 inorganic component content types obtained by ICP-MS[1], we classified the products according to seven product types, fourteen product parts, and nine cooking methods.

\subsection{Data preparation considering ICP-MS analysis er-}

ror Data was prepared considering analysis errors for the 11 inorganic components that can be quantified by ICPMS. Therefore, expected measurement errors for the 88 pork products analyzed by ICP-MS were also considered.

The assumed measurement error for the 11 inorganic component types is $\pm 10 \%$. Thereafter, classification and collation into each category (product type, product part, cooking method) was carried out using a neural network for

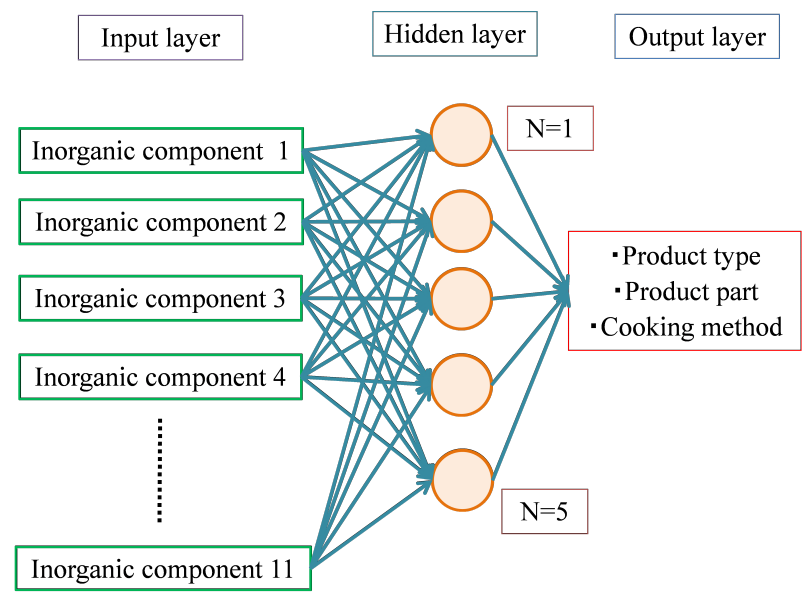

Figure 1: Image of neural network using inorganic components. 
Table 1: Each pork product category.

\begin{tabular}{lll}
\hline Product type & Product part & Cooking method \\
\hline Red meat & Picnic shoulder & Roasted \\
Without subcutaneous fat & Picnic shoulder loin & Boiled \\
Lean and fat & Outside ham & Raw \\
Fatty meat & Ham & Smoked \\
Offal and byproducts & Loin & Paste \\
Ground meat & Fillet & Sausage \\
Meat substitute & Belly & Ham \\
- & Ground meat & Bacon \\
- & Pig's feet & Breaded and fried pork \\
- & Visceral meat & - \\
- & Cartilage & - \\
- & Gelatin & - \\
- & Meat substitute & - \\
\hline
\end{tabular}

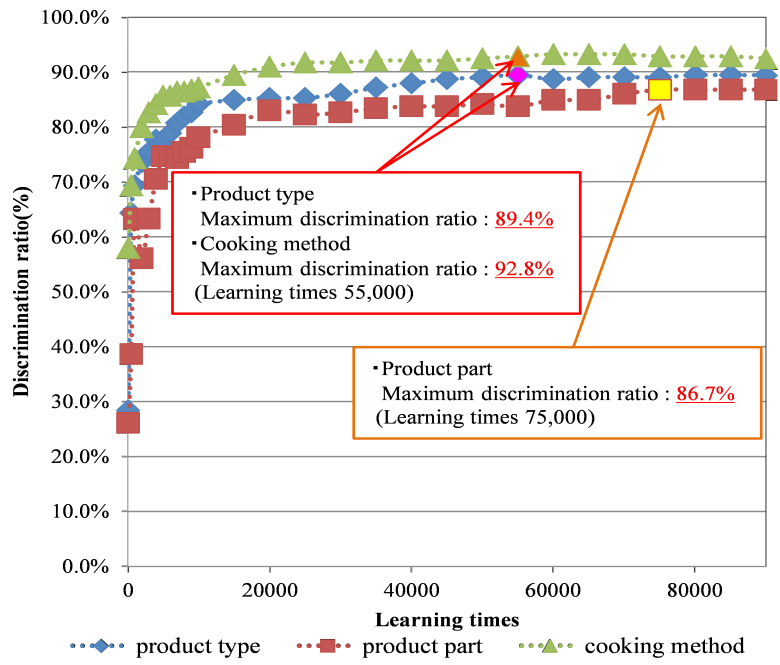

(a) Determination rate and learning iteration plot

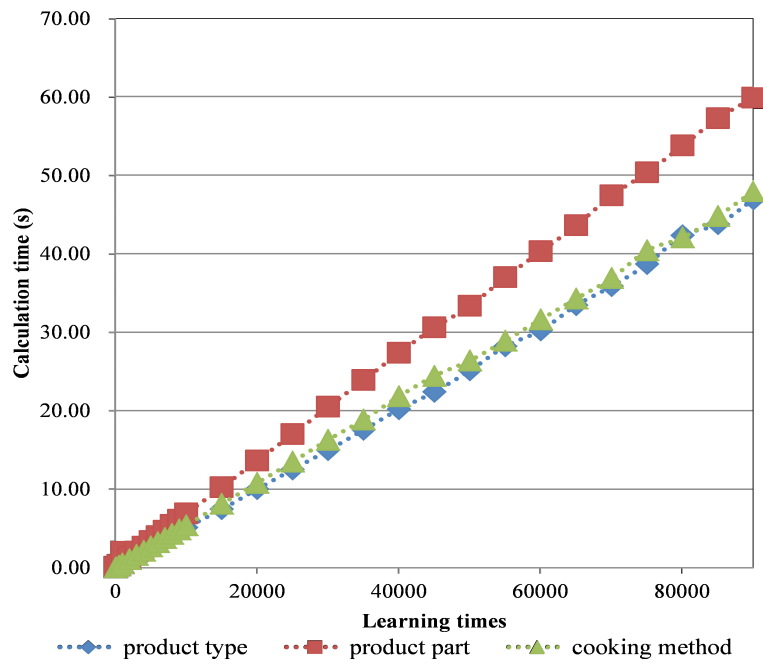

(b) Calculation time of neural network by learning iteration

Figure 2: Result of neural network analysis using inorganic component for pork products.

264 pork product types, considering errors. The contents of each category are listed in Table 1.

\subsection{Calculation of discrimination rate by neural net-} work From the 11 inorganic component types, it was determined whether the 264 pork product types could be classified into each of three categories: product type, product part, and cooking method by the neural network calculated discrimination rate.

A neural network is a mathematical model that expresses neurons and their connections in the human brain. It consists of input, output, and hidden layers [14] [15]. The information accepted at the input layer is judged at the output layer, and information conforming to each category is the output (Fig. 1). Among them, to prescribe the output value at output time, a hidden differentiable activating function layer is used.

For the calculation, we used the Weka statistical analysis software (The University of Waikato) with Windows 10 OS, 8 GB memory, and Intel Core i5-8500 processor.

The discrimination ratio is determined by Eq. 1 below. This expression is obtained by dividing the number of samples correctly predicted for each category defined in the neural network by the total sample number, 264. In this report, the results set with five middle layers, and zero to 90,000 learning iterations, are shown.

Determination rate $(\%)$

$$
=\frac{\text { Positive discrim samples }}{\text { All samples }} \times 100
$$

\section{Result and discussion}

Figure 2 shows the neural network analysis results for each of the three categories using inorganic component contents contained in all pork products. 
Table 2: Neural network analysis result by product type.

\begin{tabular}{lcccc}
\hline Product type & True & False & False rate & True rate \\
\hline Red meat & 36 & 3 & $7.7 \%$ & $92.3 \%$ \\
$\begin{array}{l}\text { Without } \\
\text { subcutaneous fat }\end{array}$ & 21 & 15 & $41.7 \%$ & $58.3 \%$ \\
Lean and fat & 36 & 9 & $20.0 \%$ & $80.0 \%$ \\
Fatty meat & 30 & 0 & $0.0 \%$ & $100.0 \%$ \\
Offal and & 33 & 0 & $0.0 \%$ & $100.0 \%$ \\
byproducts & 5 & 1 & $16.7 \%$ & $83.3 \%$ \\
Ground meat & 75 & 0 & $0.0 \%$ & $100.0 \%$ \\
Meat substitute & 236 & 28 & $10.6 \%$ & $89.4 \%$ \\
\hline Total & & & & \\
\hline
\end{tabular}

Table 3: Neural network analysis result by product part.

\begin{tabular}{lcccc}
\hline Product part & True & False & False rate & True rate \\
\hline Picnic shoulder & 15 & 9 & $37.5 \%$ & $62.5 \%$ \\
Picnic shoulder loin & 21 & 3 & $12.5 \%$ & $87.5 \%$ \\
Outside ham & 12 & 12 & $50.0 \%$ & $50.0 \%$ \\
Ham & 22 & 8 & $26.7 \%$ & $73.3 \%$ \\
Loin & 32 & 1 & $3.0 \%$ & $97.0 \%$ \\
Fillet & 11 & 1 & $8.3 \%$ & $91.7 \%$ \\
Belly & 9 & 0 & $0.0 \%$ & $100.0 \%$ \\
Ground meat & 6 & 0 & $0.0 \%$ & $100.0 \%$ \\
Pig's feet & 3 & 0 & $0.0 \%$ & $100.0 \%$ \\
Visceral meat & 24 & 0 & $0.0 \%$ & $100.0 \%$ \\
Cartilage & 3 & 0 & $0.0 \%$ & $100.0 \%$ \\
Gelatin & 2 & 1 & $33.3 \%$ & $66.7 \%$ \\
Meat substitute & 69 & 0 & $0.0 \%$ & $100.0 \%$ \\
Total & 229 & 35 & $13.3 \%$ & $86.7 \%$ \\
\hline
\end{tabular}

Table 4: Neural network analysis result by cooking method.

\begin{tabular}{lcccc}
\hline Cooking method & True & False & False rate & True rate \\
\hline Roasted & 11 & 7 & $38.9 \%$ & $61.1 \%$ \\
Boiled & 20 & 1 & $4.8 \%$ & $95.2 \%$ \\
Raw & 153 & 0 & $0.0 \%$ & $100.0 \%$ \\
Smoked & 2 & 1 & $33.3 \%$ & $66.7 \%$ \\
Paste & 2 & 1 & $33.3 \%$ & $66.7 \%$ \\
Sausage & 27 & 0 & $0.0 \%$ & $100.0 \%$ \\
Ham & 24 & 0 & $0.0 \%$ & $100.0 \%$ \\
Bacon & 2 & 7 & $77.8 \%$ & $22.2 \%$ \\
Breaded and fried & 6 & 0 & $0.0 \%$ & $100.0 \%$ \\
pork & 247 & 17 & $6.4 \%$ & $93.6 \%$ \\
Total & & & &
\end{tabular}

As shown in Fig. 2(a), the classification and collation of 264 pork products to product type caused the discrimina- tion ratio to improve as the number of learning iterations increased. The highest discrimination ratio for product type, product parts, and cooking method was $89.4 \%, 86.7 \%$, and $92.8 \%$ at 55,000, 75,000, and 55,000 learning iterations, respectively. After the maximum discrimination ratio was reached, no improvement in discrimination ratio was obtained with an increase in the learning iterations in any of the categories. For product part categories having more classification items, compared to product type and cooking method, the discrimination rate was lower and learning iterations needed were higher. It is conceivable that this correspondence to the classification item, which involved classification and collation by 11 inorganic components, was complicated by calculation.

As shown in Fig. 2(b), it was confirmed that the calculation time showed a positive correlation with learning iterations and that more calculation time was required as learning iterations increased. The trend of calculation time with respect to the number of learning iterations for each category showed similar calculation time for product type and cooking method; however, it confirmed that product part required more time compared to them.

Table 2 shows the correct discrimination ratio by product type. Consequently, fatty meat, offal and byproducts, and meat substitute had a $100 \%$ discrimination rate, while meat without subcutaneous fat showed a low value of $58.3 \%$. Lean and fat meat and ground meat are also $80.0 \%$ or more, but about $20 \%$ misidentification was confirmed. This is because meat without subcutaneous fat, lean and fat meat, and ground meat contain fatty meat, and because the composition of inorganic components per $100 \mathrm{~g}$ of product is similar, higher misidentification rates are expected.

Table 3 shows the correct discrimination rate for product parts. Loin, fillet, belly, ground meat, pig's feet, visceral meat, cartilage, and meat substitute show high value of $91.7-100 \%$ for the normal discrimination rate, which indicates that the inorganic components can be classified and verified with high accuracy by neural network learning.

However, because picnic shoulder, picnic shoulder loin, outside ham, and ham are portions that are close to each other, the discrimination accuracy is lower than that of the others, between $50.0-87.5 \%$.

Gelatin has a correct discrimination rate of $66.7 \%$, which is an erroneously discriminated factor, as pig's feet also contains gelatin.

Table 4 shows the correct discrimination rate by cooking method. Boiled, raw, sausage, ham, \& breaded and fried pork showed high accuracy of 95.2-100\%.

However, the accuracy of roasted, smoked, and paste was low and between $61.1-66.7 \%$, with bacon's at the lowest of $22.2 \%$.

The results show that improving discrimination accuracy of alike parts is problematic; however, different parts can be classified and verified with high accuracy.

It was shown that pork products classification into categories can be performed and verified with inorganic components measured by ICP-MS. However, because an overall discrimination rate of $90 \%$ cannot be achieved outside of 
the cooking method category, improving the further classification collation accuracy is a problem for further study.

\section{Conclusions}

In this report, we presented the possibility of classification collation of pork products using inorganic components that can be quantified by ICP-MS as a preliminary report. In the future, we will examine the hidden layer and learning iterations to improve the classification matching accuracy of each category, and we will actually determine to which category the product with unknown classification belongs.

\section{Acknowledgment}

This research was partially supported by the Ministry of Education, Science, Sports and Culture, Grant-in-Aid for Scientific Research(C), 2015-2019(15K00806, Yoichiro Kojima).

\section{References}

[1] Ministry of Education, Culture, Sports, Science and Technology: Food ingredient database, https://fooddb.mext. go.jp/, access date: 2018.10 (In Japanese).

[2] Japan Food Research Laboratories, "Component Analysis Point for Nutrition Labeling", Central Regulation, pp.116140, 2007(In Japanese).

[3] Okiya Akihiro, "Determinants of the Deliciousness of Meat", Japanese Nutrition Journal, Vol.60, No.3, pp.119129, 2002(In Japanese).

[4] Yoichiro Kojima, "Fundamental study on classification of commercial salt for various analytical value", The Japanese journal of taste and smell research, Vol.15, No.3, pp.393396, 2008(In Japanese).

[5] Yoichiro Kojima and Tsuyoshi Mikami, "Development of taste sensor using instrumental analyses and information processing technique", The Japanese journal of taste and smell research, Vol.16, No.3, pp.513-516, 2009(In Japanese).

[6] Yuta Kimura, Yoichiro Kojima, Tsuyoshi Mikami and Kuniharu Utsuno, "Fundamental study on classification of beef for development of online taste sensing system", The Japanese journal of taste and smell research, Vol.17, No.3, pp.405-408, 2010(In Japanese).

[7] Yuta Kimura, Yoichiro Kojima, Tsuyoshi Mikami and Kuniharu Utsuno, "Classification of beef using food composition data and discriminate analysis", The Japanese journal of taste and smell research, Vol.18, No.3, pp.443-446, 2011(In Japanese).

[8] Motoki Goda, Yoichiro Kojima, Tsuyoshi Mikami and Kuniharu Utsuno, "Study on classification of pork using inorganic analytical value and cluster analysis", The Japanese journal of taste and smell research, Vol.18, No.3, pp.447450, 2011(In Japanese).

[9] Yu Miyake, Yoichiro Kojima, Tsuyoshi Mikami and Kuniharu Utsuno, "Discrimination of salt by the various data and multivariate analysis", The Japanese journal of taste and smell research, Vol.19, No.3, pp.417-420, 2012(In Japanese).

[10] Motoki Goda, Yoichiro Kojima, Tsuyoshi Mikami, Kuniharu Utsuno and and Syunsuke Iwanami, "Classification of pork products by inorganic content and multivariate analysis", The Japanese journal of taste and smell research, Vol.18, No.3, pp.451-454, 2011(In Japanese).

[11] Hiroki Nakajima, Motoki Goda, Yoichiro Kojima, Tsuyoshi Mikami and Syunsuke Iwanami, "Classification of the food using nutrition information and chemometrics", The Japanese journal of taste and smell research, Vol.18, No.3, pp.347-350, 2013(In Japanese).

[12] Motoki Goda and Yoichiro Kojima, "Study on Classification Possibility of Pork Products by Chemometrics", The Japanese Journal of the Institute of Industrial Applications Engineers, Vol.6, No.1, pp.33-43, 2018(In Japanese).

[13] Motoki Goda and Yoichiro Kojima, "Fundamental study on food classification technique by multivariate data and the physicochemical analysis", The Japanese journal of taste and smell research, Vol.21, No.3, pp.399-402, 2014(In Japanese).

[14] W. S. McCulloch and W. Pitts, "A logical calculus of the ideas immanent in neurons activity", Bulletin of Mathematical Biology, Vol.5, pp.115-133, 1943.

[15] Ling Zhang and Bo Zhang, "Geometrical representation of McCulloch-Pitts neural model and its applications", IEEE Transactions on Neural Networks, Vol.10, No.4, pp.925-929, 1999. DOI: $10.1109 / 72.774263$

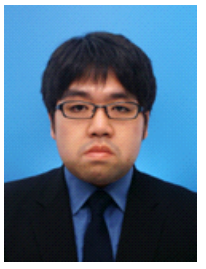

Goda Motoki (Member) In March 2015, completed the master's program at Hokkaido University Graduate School of Environmental Science, and received a master's degree in environmental science. The same year, he joined North Japan Port Consultants Co., Ltd. and was assigned to the 1st Engineering Department Planning Team. In April 2017, appointed as a researcher at Hokkaido University of science. Engaged in research for port planning, behavior factor analysis, food quality control using AI / IoT and chemical component value information, and identification of deliciousness factors

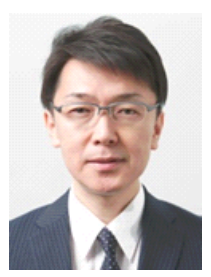

Kojima Yohichiro (Member) He received his BE, ME and Dr. Eng. degree, all in applied electronics engineering, from Hokkaido Institute of Technology in 1990, 1992 and 1995, respectively. He is now a Professor at Department of Electrical and Electronic Engineering, Faculty of Engineering of Hokkaido University of Science. His main research interests are focused on development of taste sensing. Dr. Kojima is a member of the Institute of Industrial Applications Engineers. 